ARTICLE

\title{
Two distinctive energy migration pathways of monolayer molecules on metal nanoparticle surfaces
}

Jiebo Li,2, Huifeng Qian ${ }^{3}$, Hailong Chen², Zhun Zhao ${ }^{3}$, Kaijun Yuan ${ }^{4}$, Guangxu Chen ${ }^{5}$, Andrea Miranda², Xunmin Guo ${ }^{2}$, Yajing Chen ${ }^{4}$, Nanfeng Zheng ${ }^{5}$, Michael S. Wong ${ }^{3,2} \&$ Junrong Zheng ${ }^{1,2}$

Energy migrations at metal nanomaterial surfaces are fundamentally important to heterogeneous reactions. Here we report two distinctive energy migration pathways of monolayer adsorbate molecules on differently sized metal nanoparticle surfaces investigated with ultrafast vibrational spectroscopy. On a $5 \mathrm{~nm}$ platinum particle, within a few picoseconds the vibrational energy of a carbon monoxide adsorbate rapidly dissipates into the particle through electron/hole pair excitations, generating heat that quickly migrates on surface. In contrast, the lack of vibration-electron coupling on approximately $1 \mathrm{~nm}$ particles results in vibrational energy migration among adsorbates that occurs on a twenty times slower timescale. Further investigations reveal that the rapid carbon monoxide energy relaxation is also affected by the adsorption sites and the nature of the metal but to a lesser extent. These findings reflect the dependence of electron/vibration coupling on the metallic nature, size and surface site of nanoparticles and its significance in mediating energy relaxations and migrations on nanoparticle surfaces.

\footnotetext{
${ }^{1}$ College of Chemistry and Molecular Engineering, Beijing National Laboratory for Molecular Sciences, Peking University, Beijing 100871, China. ${ }^{2}$ Department of Chemistry, Rice University, 6100 Main Street, Houston, Texas 77005, USA. ${ }^{3}$ Department of Chemical and Biomolecular Engineering, Rice University, 6100 Main Street, Houston, Texas 77005, USA. ${ }^{4}$ State Key Laboratory of Molecular Reaction Dynamics, Dalian Institute of the Chemical Physics, Chinese Academy of Sciences, Dalian, Liaoning 116023, China. ${ }^{5}$ College of Chemistry and Chemical Engineering, Xiamen University, Xiamen, Fujian 361005, China. Correspondence and requests for materials should be addressed to J.Z. (email: zhengjunrong@gmail.com).
} 
$\mathrm{O}$ $\mathrm{n}$ the surfaces of heterogeneous catalysts, energy dissipation and migration dynamics determine the reaction local temperature and the ability of an adsorbate to cross the reaction barrier. Knowledge about the energy dynamics is essential for a thorough understanding of the selectivity and kinetics of catalytic reactions-and indispensable for developing rational guiding theories of catalyst design ${ }^{1-3}$. However, to experimentally investigate and theoretically describe energy dissipation and migration mechanisms on the surface of a metal nanoparticle, a key component of many important heterogeneous catalysts, remains a grand challenge ${ }^{1,4-10}$. Typically, theoretically modelling a reaction requires modifications of the nuclear potential energy surface with the assumption of separated nuclear and electronic motions during the nuclear movements across its transition state ${ }^{11,12}$. This approach, known as the Born-Oppenheimer approximation, which serves as the cornerstone of chemical kinetic theory, allows the electronic energy to be considered independent of the nuclear kinetic energy. Based on the Born-Oppenheimer approximation, the energy $(<0.5 \mathrm{eV})$ released in chemisorption or physisorption of molecules on metal surfaces was usually believed to be dissipated by surface vibrations (phonons), and electronic excitations through electron/vibration coupling were neglected ${ }^{13-16}$. However, some experimental evidence . $^{17-22}$ and theoretical studies ${ }^{23}$ have shown the nonadiabatic effects of vibration/electron coupling on flat metal and some metal nanoparticle surfaces ${ }^{10,24}$, questioning the general applicability of the approach. This problem is even more complicated on the surfaces of catalytic metal nanoparticles, as the electronic properties can vary with particle size ${ }^{25}$ and surface sites ${ }^{26}$.

In this work, we demonstrate that the real-time energy migration dynamics between molecules on different surface sites of metal nanoparticles can be monitored with an ultrafast multiple dimensional vibrational spectroscopy. Two distinctive energy pathways are observed for a monolayer of carbon monoxide (CO) molecules adsorbed on different surface sites of a series of platinum $(\mathrm{Pt})$ nanoparticles. On the surface of a $5 \mathrm{~nm}$ particle, which is metallic and traditionally theorized to exhibit electron/vibrational coupling ${ }^{27}$, the energy migrations among different sites are very fast, occurring within a few picoseconds. In contrast, the energy migration dynamics between surface sites of small, semi-conductive Pt nanoparticles ( $\sim 1 \mathrm{~nm}$ clusters) with a much smaller degree of electron/vibrational coupling are more than 1 order of magnitude slower. Further investigations reveal a similar particle-size dependence for the vibrational energy relaxations of the $\mathrm{CO}$ molecules: the vibrational relaxations on larger particles $(2-11 \mathrm{~nm})$ are also more than 1 order of magnitude faster than those on the $1 \mathrm{~nm}$ clusters. Changing the surface adsorption sites and the nature of the metal from $\mathrm{Pt}$ to Palladium (Pd) also alters the fast energy relaxation but to a lesser extent compared with the size effect.

\section{Results}

CO molecules on different surface sites of Pt particles. The samples investigated in this work are 1 (Fig. 1a), 2, 5 (Fig. 1b) and $11 \mathrm{~nm}$ Pt clusters and nanoparticles and $\sim 1$ and $5 \mathrm{~nm}$ Pd clusters and particles, all of which are coated with a monolayer of $\mathrm{CO}$ and ligand molecules (particle characterizations are in Supplementary Figs 1-5). Pt and Pd nanoparticles are important metals in the field of catalysis. They have been used to catalyse the oxidation of $\mathrm{CO}$, aiding in the removal of automobile exhausts, and also serve as classical model systems for mechanistic studies of heterogeneous catalysis for many years ${ }^{28,29}$. On the surface of the triangular prismatic $1 \mathrm{~nm}$ particle, $\mathrm{CO}$ molecules can reside on either the atop site (I) on the top of a Pt atom or the bridge site
(II) between two Pt atoms (Fig. 1a). The $\mathrm{CO}$ molecules on the atop sites have a $\mathrm{CO}$ stretch $0-1$ transition frequency at $\sim 2,047 \mathrm{~cm}^{-1}$, and those at the bridge sites have a frequency at $\sim 1,860 \mathrm{~cm}^{-1}$ (Fig. 1c (black curve)) $)^{30}$. On the surface of $5 \mathrm{~nm}$ polyhedral-like $\mathrm{Pt}$ nanoparticles (Fig. 1b), CO molecules can occupy the terrace atop site (III), the step atop site (I) and the bridge site $(\mathrm{II})^{31,32}$. As displayed in the two-dimensional infrared (2D IR) spectrum in Fig. 1d, CO molecules on site III have a CO stretch $0-1$ transition frequency $\left(\omega_{3}\right.$, probe frequency) at $\sim 2,113 \mathrm{~cm}^{-1}$. The frequency on site I is $\sim 2,053 \mathrm{~cm}^{-1}$, and that on site II is $\sim 1,880 \mathrm{~cm}^{-1}$ (Fig. 1c). The blue peaks underneath the red peaks along the $\omega_{3}$ axis in Fig. 1d are the CO stretch 1-2 transition peaks. The blue peaks have lower $\omega_{3}$ frequencies than the corresponding red peaks because of vibrational anharmonicities ${ }^{33-35}$. On the surfaces of other $\mathrm{Pt}$ particles ( 2 and $11 \mathrm{~nm}$ ), CO molecules can also occupy the atop, bridge and terrace sites. Because the surface structures and the relative surface-site numbers differ on particles of different sizes, the frequency and relative intensity of the $\mathrm{CO}$ vibrational peak on each surface site vary with the particle size (Fig. 1c).

Two distinctive energy migration pathways. We employed two experimental strategies to investigate the energy migration pathways on the surfaces of these Pt nanoparticles by the realtime detection of (a) heat/vibrational energy migration on the nanoparticle surfaces and (b) direct measurements of the $\mathrm{CO}$ stretch first excited state population relaxations at different surface sites under various conditions. In doing so, we applied an ultrafast frequency-resolved mid-IR pulse to excite $\mathrm{CO}$ molecules on each site to the first vibrational excited state. Another ultrafast mid-IR pulse that covers a frequency range $^{36}$ from $\sim 1,000$ to $\sim 3,500 \mathrm{~cm}^{-1}$ simultaneously detects the optical responses at the exciting frequencies, as well as frequencies associated with molecules on other surface sites. Tuning the waiting time between the excitation and detection pulses, (a) the heat generation was monitored by recording the heat-induced $\mathrm{CO}$ stretch 0-1 transition bleaching signals of CO molecules and (b) the direct $\mathrm{CO}$ stretch excitation relaxations were monitored in real-time by measuring the decays of the CO stretch $0-1$ and 1-2 transition signals. (Signal origins are presented in Supplementary Method).

As displayed in Fig. 2a (where only the $0-1$ transition signal is plotted), on the surface of the $5 \mathrm{~nm} \mathrm{Pt}$, after the $\mathrm{CO}$ molecules on the bridge site II are initially excited by a laser pulse, at zero waiting time, there is only one diagonal peak (peak 1) in the $0 \mathrm{ps}$ spectrum. At 2 ps, a cross peak (peak 2) grows in at $2,062 \mathrm{~cm}^{-1}$, $\sim 9 \mathrm{~cm}^{-1}$ higher in frequency than that of the CO $0-1$ transition on site I (Fig. 2b). At 5 ps, peak 2 grows in further and surpasses peak 1 in intensity. Depiction of 2D IR approach of the $5 \mathrm{~nm} \mathrm{Pt}$ sample is shown in Supplementary Fig. 6. In contrast, the growth of the cross peak $2^{\prime}$ on the surface of the $1 \mathrm{~nm} \mathrm{Pt}$ particles is significantly slower (Fig. 2c,d). At a waiting time of $50 \mathrm{ps}$, its intensity is still less than $20 \%$ of the diagonal peak $1^{\prime}$ (Fig. 2d). The growth of peaks 2 and 3 (Fig. 2b) is caused by the heat generated from the vibrational relaxation of the $\mathrm{CO}$ stretch on site II, similar to those in liquid samples described in great detail in our previous work ${ }^{36,37}$. The growth of peaks $2^{\prime}$ and $6^{\prime}$ (Fig. $2 \mathrm{~d}$ ) is caused by vibrational energy exchanging between two sites. Illustration of 2D IR approach on the $1 \mathrm{~nm}$ Pt sample is shown in Supplementary Fig. 7.

The particle size-dependent responses of the step site CO indicate different energy migration pathways. Recent work ${ }^{25,38}$ suggests that the electronic properties of metal nanoparticles are dictated by the number of atoms and thus the particle size. For example, $5 \mathrm{~nm}$ polyhedral particle is metallic ${ }^{39,40}$ and exhibits a 
a

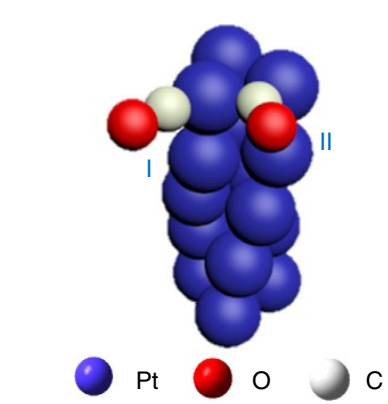

C

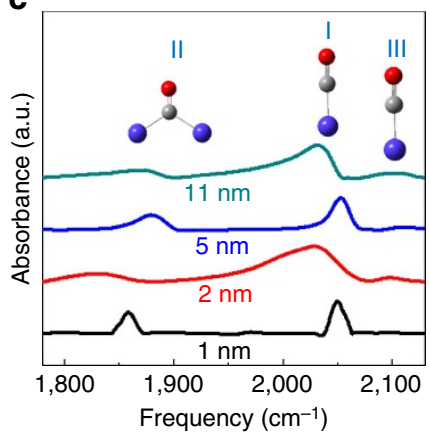

b

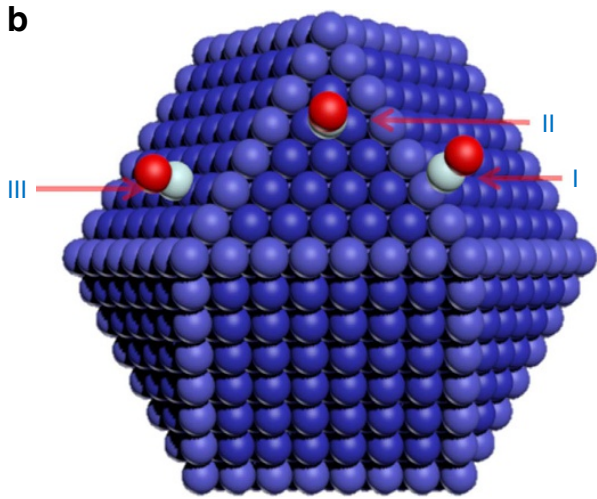

d

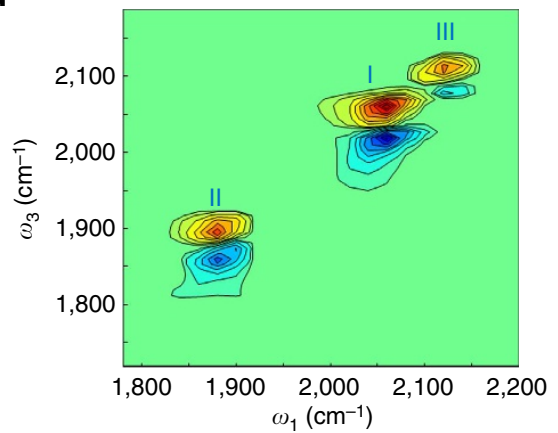

Figure 1 | Illustration and spectrums of $\mathbf{C O}$ molecules on Pt particles. (a) Illustrated CO molecules on the atop (I) and bridge (II) sites of a $1 \mathrm{~nm}$ Pt nanoparticle $\left(\mathrm{Pt}_{15}\right)$. In the sample, the actual CO coverage on both surface sites is $100 \%$. (b) Illustrated CO molecules on the step (I), bridge (II) and terrace (III) sites of a $5 \mathrm{~nm}$ Pt nanoparticle. In the sample, the actual CO coverage on both surface sites is saturated. (c) FTIR spectra of CO molecules on Pt nanoparticles in the frequency range of $C \equiv O$ stretch 0-1 transition. (d) 2D IR spectrum of CO molecules on $5 \mathrm{~nm}$ Pt nanoparticles at zero waiting time, clearly showing the three different CO species ( $y$-axis is probe frequency, and $x$-axis is pump frequency). The intensities of peaks II and III and their corresponding blue peaks along the $y$-axis are magnified by 13 and 22 times, respectively, to be comparable to that of peak I.

continuum of electronic states. As a result, theoretical studies predict that the conduction electrons on these metallic $\mathrm{Pt}$ nanoparticle surfaces can be excited from just below to just above the Fermi level (excitation of an electron/hole pair) by the resonant energy transfer from the $\mathrm{CO}$ stretch vibrational excitation $^{27}$. The electron/hole pairs excited by the vibrational relaxation can quickly recombine and release their energy to the lattice motions (phonons, or 'heat') within $1 \sim 2 \mathrm{ps,} \mathrm{and} \mathrm{quickly}$ migrate on the surface, raising the metal surface temperature ${ }^{41,42}$. As a result of the temperature increase, the CO stretch 0-1 transition frequency $\left(2,053 \mathrm{~cm}^{-1}\right)$ on site I red-shifts, which is corroborated by the temperature-dependent Fourier transform infrared spectroscopy (FTIR) spectra (in Supplementary Fig. 8). Such a red shift produces a bleaching signal at a frequency $\left(2,062 \mathrm{~cm}^{-1}\right)$ higher than $2,053 \mathrm{~cm}^{-1}$ and a new absorption at a frequency $\left(2,047 \mathrm{~cm}^{-1}\right)$ lower than $2,053 \mathrm{~cm}^{-1}$ in the $2 \mathrm{D}$ IR spectra (Fig. 2b). The excitation frequency $\left(\omega_{1}=1,880 \mathrm{~cm}^{-1}\right)$ of peaks 2 and 3 reflects the origin (site II) of the energy dissipation process, and the respective detection frequencies $\left(\omega_{3}=2,062\right.$ and $2,047 \mathrm{~cm}^{-1}$ ) demonstrate the spectral changes of the CO stretch on site I caused by the process. Therefore, how the intensities of the two peaks change with the waiting time is indicative of thermal energy migration dynamics from site II to site I. As displayed in Fig. 3a, peak 2 grows very fast and reaches a maximum at $2 \sim 3$ ps and then decays slowly, suggesting that the thermal energy migrates from site II to site I very rapidly within $2 \sim 3$ ps and then dissipate to environment slowly. By comparing the 2D IR results to the temperature difference FTIR spectra (Fig. 3b), the temperature increase on site II within the initial $2 \sim 3$ ps is found to be $>57 \mathrm{~K}$ (Fig. $3 \mathrm{~b}$ ), and the increase drops to about $25 \mathrm{~K}$ at a delay of $25 \mathrm{ps}$ after the thermal energy has further dissipated to the environment. The spectral changes caused by the temperature increase are very small in the frequency range of bridge site CO stretch $\left(\sim 1,880 \mathrm{~cm}^{-1}\right)$ (Supplementary Fig. $8 \mathrm{~b}$ ). The additional overlap, occurring with the tail of a very strong 12 absorption signal of the step atop site CO stretch after 2 ps (Fig. 2b), makes the heat-induced cross peaks around 6 and 7 $\left(\omega_{1}=2,053 \mathrm{~cm}^{-1}, \omega_{3}=1,880 \mathrm{~cm}^{-1}\right)$ too weak to be observed.

In contrast to peaks 2 and 3 of the $5 \mathrm{~nm}$ Pt particle, the growth of peaks $2^{\prime}$ and $3^{\prime}$ are not caused by the migration of thermal energy generated from vibrational relaxation, but by the direct vibrational energy exchange between sites I and II CO as their frequencies are identical to those of the diagonal peaks $4^{\prime}$ and $5^{\prime}$ (Fig. 2d). The growth of the cross peaks is not caused by chemical exchange, in which case the peak growth rate constant ratio of $6^{\prime}$ over $2^{\prime}$ would be equal to 1 (ref. 35). The $1 \mathrm{~nm}$ cluster $\left(\mathrm{H}_{2}\left[\mathrm{Pt}_{3}(\mathrm{CO})_{3}\left(\mu_{2}-\mathrm{CO}\right)_{3}\right]_{5}\right)$ is a semiconductor with a HOMOLUMO energy gap of $\sim 1.7 \mathrm{eV}$ (see visible spectrum in Supplementary Fig. 1d $)^{43,44}$. This energy splitting is considerably larger than the $\mathrm{CO}$ vibrational $0-1$ transition energy of $0.25 \mathrm{eV}$, and thus the $\mathrm{CO}$ stretch vibrational energy cannot excite the Pt electrons from the HOMO to the LUMO. Therefore, the CO vibrational energy on $1 \mathrm{~nm}$ sample cannot be mediated by electronic excitation, it can only transfer directly to the bridge site (II) C-O stretch (peaks $2^{\prime}$ and $6^{\prime}$ ) via vibration/vibration coupling. When direct vibrational energy exchange occurs, the population ratio is determined by detailed balance (this system is $\left.e^{\frac{2,047 \mathrm{~cm}^{-1}-1,860 \mathrm{~cm}^{-1}}{200 \mathrm{~cm}^{-1}}}=2.5\right)^{45}$. Kinetic analyses show that the peak growth rate ratio (Fig. 3c) is also 2.5 (detailed kinetic analyses are found in Supplementary Note 1). No heat signal is observed up to 50 ps (Fig. 2d) (The control 
a

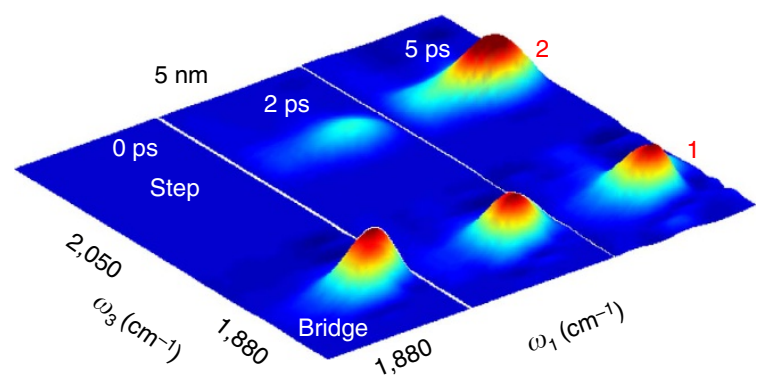

C

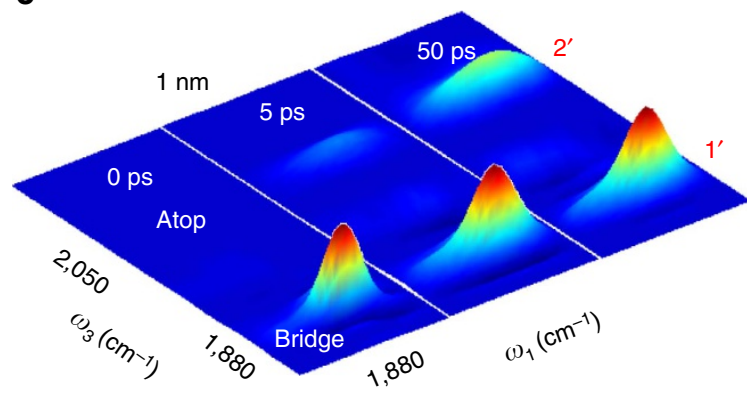

b
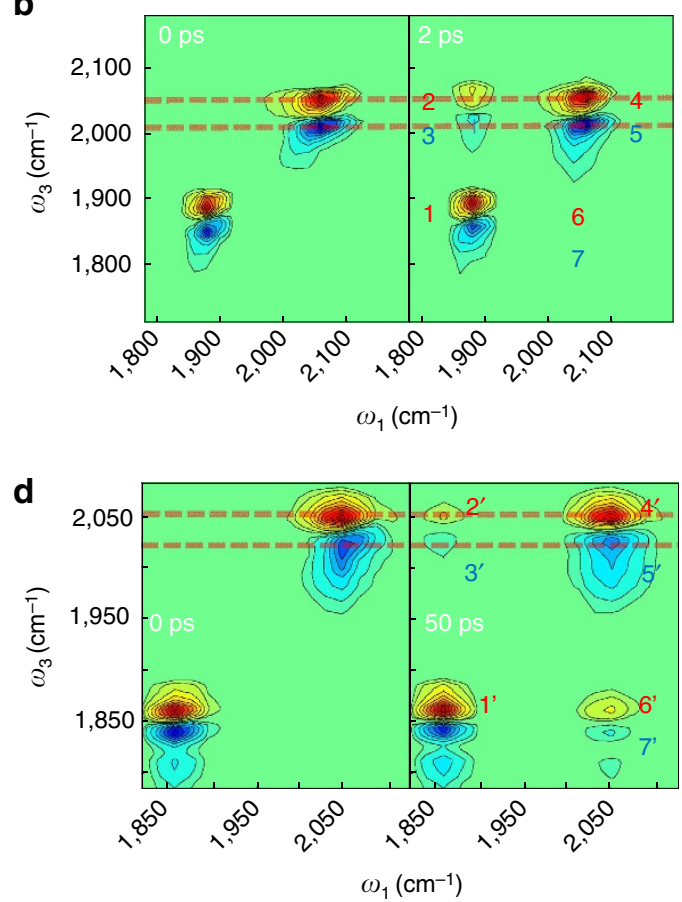

Figure 2 | Two distinctive pathways for vibrational energy generation. (a) 2D IR spectra of CO stretch ( $0-1$ transition) on $5 \mathrm{~nm}$ Pt particles at three waiting times after exciting $\mathrm{CO}$ on the bridge site and detecting responses on both bridge and step atop sites. The growth of peak 2 at the step site indicates the generation of heat. (b) 2D IR spectra of CO stretch (both 0-1 and 1-2 transitions) on $5 \mathrm{~nm}$ Pt particles at two waiting times. The frequencies of the cross peak pairs (peaks 2 and 3) are different from those of the diagonal peak pair (peaks 4 and 5), indicating that peaks 2 and 3 arise because of heat generation. Red dashed lines are drawn to highlight the frequency differences. (c) 2D IR spectra of CO stretch (0-1 transition) on $1 \mathrm{~nm} P t$ particles at three waiting times by exciting $\mathrm{CO}$ on the bridge site and detecting responses on both bridge and step atop sites. The growth of the peak $2^{\prime}$ at the step site indicates the direct vibrational exchange between CO molecules on the two surface sites. (d) 2D IR spectra of CO stretch (both 0-1 and 1-2 transitions) on $1 \mathrm{~nm}$ Pt particles at two waiting times. The frequencies of the cross peak pairs (peaks $2^{\prime}$ and $3^{\prime}$ ) are the same as those of the diagonal peak pair (peaks $4^{\prime}$ and $5^{\prime}$ ), indicating that peaks $2^{\prime}$ and $3^{\prime}$ are due to direct vibrational transfer between $C O$ molecules on the two sites. The doublet of the blue peak of bridge $\mathrm{CO}$ is attributed to a Fermi resonance ${ }^{57}$. (The diagonal red peaks 1 and $1^{\prime}$ are normalized to 1 ). For the $2 \mathrm{D}$ IR spectra, $y$-axis is probe frequency, and $x$-axis is pump frequency.

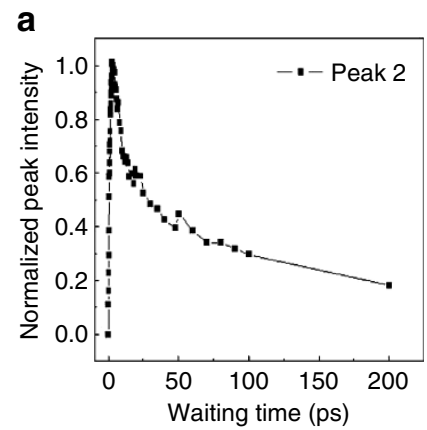

b

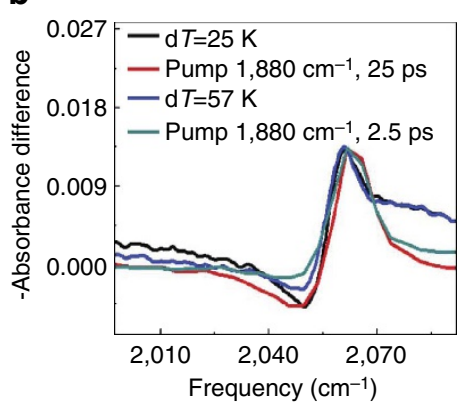

C

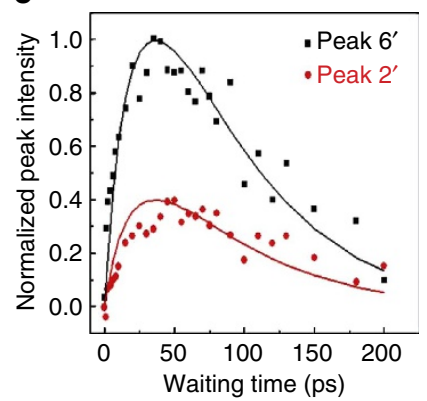

Figure 3 | Energy migration induced time-dependent spectrum dynamics. (a) The time-dependent intensities of peak $2\left(\omega_{1}=1,880 \mathrm{~cm}^{-1}\right.$, $\omega_{3}=2,062 \mathrm{~cm}^{-1}$ ) on the $5 \mathrm{~nm}$ Pt nanoparticle. (b) Comparison of temperature difference FTIR (black and blue curves) and 2D IR data at waiting times $2.5 \mathrm{ps}$ (red) and $25 \mathrm{ps}$ (cyan). Intensities were normalized to similar values for better illustration. (c) The time-dependent intensities of the cross peak signals $6^{\prime}\left(\omega_{1}=2,047 \mathrm{~cm}^{-1}, \omega_{3}=1,860 \mathrm{~cm}^{-1}\right)$ and $2^{\prime}\left(\omega_{1}=1,860 \mathrm{~cm}^{-1}, \omega_{3}=2,047 \mathrm{~cm}^{-1}\right)$ on the $1 \mathrm{~nm}$ Pt nanoparticle.

temperature-dependent FITR of $1 \mathrm{~nm}$ sample is shown in Supplementary Fig. 9). This result indicates that the heat generation induced by $\mathrm{CO}$ vibrational relaxation on the $1 \mathrm{~nm}$ Pt particle is significantly slower than $50 \mathrm{ps,} \mathrm{which} \mathrm{is} \mathrm{already}$ more than 20 times slower than on the $5 \mathrm{~nm}$ Pt particle.

Two distinctive energy dissipation pathways. The distinct energy migration pathways following the surface molecular vibrational excitations on the two sizes particles are further supported by direct measurements of the CO stretch first excited state population relaxations at different surface sites under various conditions. Figure 4 displays the waiting time-dependent CO stretch $0-1$ (red) and 1-2 (blue) transition signals of CO molecules on the step atop site of 1, 2, 5 and $11 \mathrm{~nm}$ Pt particles after excitation to the first excited state of the CO stretch. On the surface of $1 \mathrm{~nm}$ Pt particles (Fig. 4a), after $20 \mathrm{ps,} \mathrm{both} 0-1$ and 1-2 transition signals still remain $>50 \%$ of the initial intensity. However, on larger particles, the signals decay much faster. Within 4 ps more than $80 \%$ of the signal has decayed (Fig. $4 \mathrm{~b}-\mathrm{d}$ ). This demonstrates that the $\mathrm{CO}$ vibrational energy dissipates 

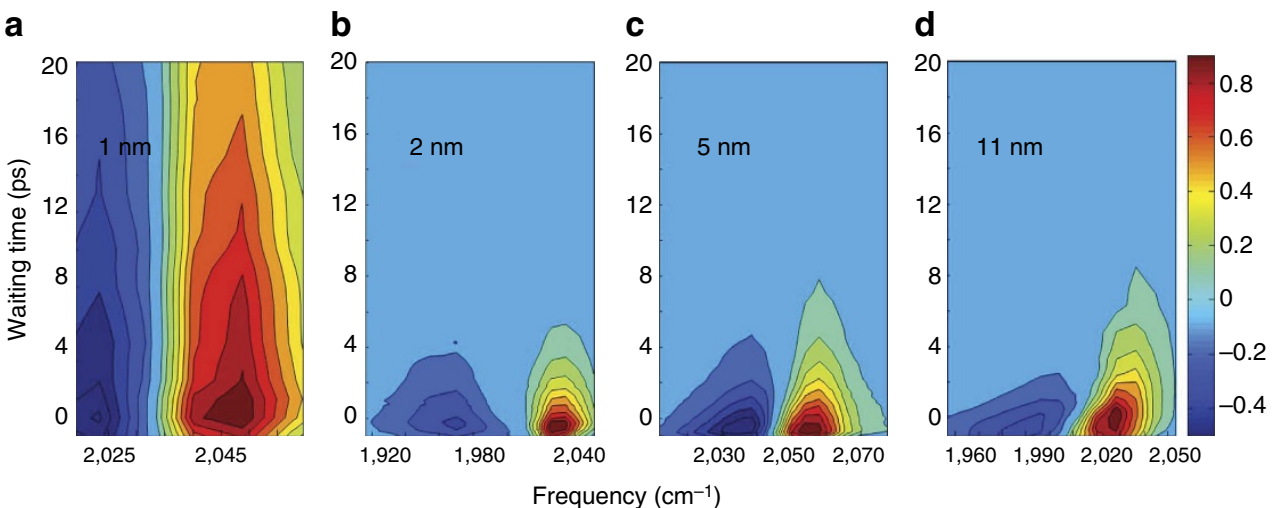

Figure 4 | Time-dependent spectrum of $\mathbf{C O}$ on Pt nanoparticles. The waiting time-dependent CO stretch 0-1 (red) and 1-2 (blue) transition signals of CO molecules on the atop step site of (a) $1 \mathrm{~nm}$ Pt particles, (b) $2 \mathrm{~nm}$ Pt particles, (c) $5 \mathrm{~nm}$ Pt nanoparticles and (d) $11 \mathrm{~nm}$ Pt nanoparticles after these molecules are excited to the $\mathrm{CO}$ stretch first excited state. The signal from the $1 \mathrm{~nm}$ particle lasts significantly longer than the signal from larger particles.

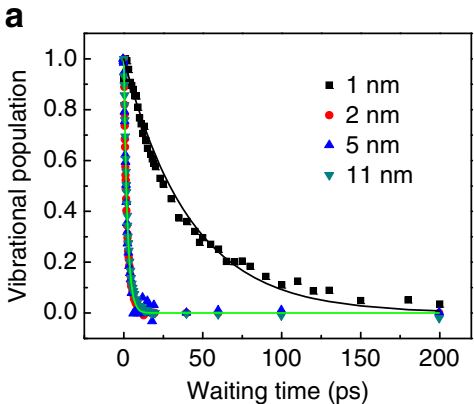

d

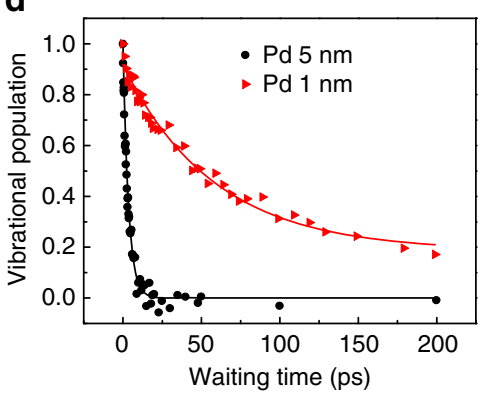

b

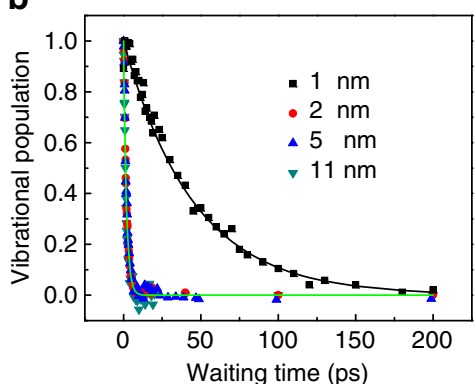

C

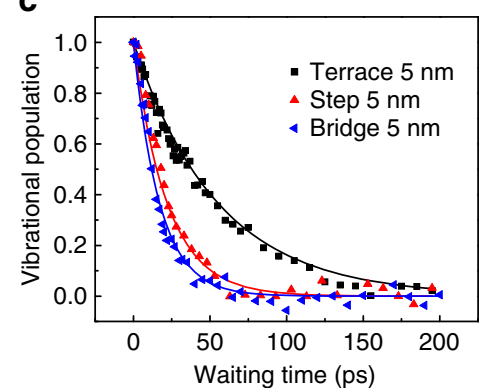

e

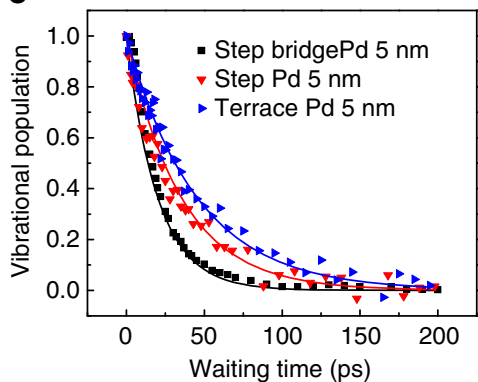

Figure 5 | Vibrational relaxation of $\mathbf{C O}$ in different conditions. (a) Vibrational relaxations of $\mathrm{CO}$ at the step site (I) on differently sized Pt particles; (b) Vibrational relaxations of $\mathrm{CO}$ at the bridge site (II) on differently sized Pt particles; (c) Vibrational relaxations of $\mathrm{CO}$ on the $5 \mathrm{~nm}$ Pt particle at different sites; (d) Vibrational relaxations of CO on the step atop site of $5 \mathrm{~nm}$ Pd particles and the atop site of $1 \mathrm{~nm} \mathrm{Pd}(\mathrm{Pd} / \mathrm{Ag})$ particles; (e) Vibrational relaxations of $\mathrm{CO}$ on $5 \mathrm{~nm}$ Pd particles at different sites. Experimental data points are fit with curves.

much faster on the surfaces of larger nanoparticles. This fast relaxation is consistent with the observed rapid heat generation from CO relaxation on step sites of metallic nanoparticles. From Fig. $4 \mathrm{~b}-\mathrm{d}$, we can also see that the $0-1$ transition signals slightly blue-shift with the increase of waiting time and last a little longer than the 1-2 transition signals, which is similar to Fig. 2c.

Quantitative analyses (Fig. 5a) of the data show that the CO stretch first excited state vibrational energy decays with a time constant of $42 \pm 5$ ps on site I of the $1 \mathrm{~nm}$ particle. In contrast, the relaxations time constants are more than 15 times faster and identical on the same site of the 3 larger-sized Pt particles, $2.2 \pm 0.2$ ps. Similarly, particle size dependence of the $\mathrm{CO}$ vibrational relaxations is observed on the bridge site (II) (Fig. 5b). The CO stretch first vibrational excited state population decays with a time constant of $47 \pm 5 \mathrm{ps}$ on the bridge site of $1 \mathrm{~nm}$ particles. On the same surface site of the larger $(2-11 \mathrm{~nm}) \mathrm{Pt}$ particles, the decay is significantly faster with a time constant of $1.8 \pm 0.3 \mathrm{ps}$, which is consistent with fast temperature rise in
Fig. 3a. In addition, the fast relaxation dynamics on step site $\mathrm{CO}$ are independent (within the experimental uncertainty $\sim 10 \%$ ) of isotopic labelling ${ }^{13} \mathrm{C}^{18} \mathrm{O}$ (Supplementary Fig. 10a ), temperature variance (293-80 K) (Supplementary Fig. 10b) and environmental modifications (Supplementary Fig. 10c). In contrast, without the surface electronic excitation, the CO relaxation on $1 \mathrm{~nm} \mathrm{Pt}$ particle surfaces follows a similar mechanism (vibration/vibration coupling) as the metal carbonyl small molecules ${ }^{46}, 1-2$ orders slower than the dynamics observed on metallic nanoparticle surfaces.

The fast CO stretch vibrational decays on metallic Pt particles surfaces $(\geq 2 \mathrm{~nm})$ were also observed on other surface sites besides sites I and II. Close examination of the rates reveals that different kinds of binding at the surface sites result in different dynamics. As shown in Fig. 5c, the $\mathrm{CO}$ vibrational energy relaxation on the terrace site (III) has a time constant $5.5 \pm 0.6 \mathrm{ps}$. The relaxations $(1.8 \pm 0.3,2.2 \pm 0.2$ and $5.5 \pm 0.6 \mathrm{ps})$ on all three surface sites are of the same order time scale and all significantly 
faster than the dynamics observed on the $1 \mathrm{~nm}$ Pt particle surface (40-50 ps). The observed metal/nonmetal dependence of $\mathrm{CO}$ relaxations is similar to that of previous experiments ${ }^{10,24}$. However, probably due to technical limitations, previous experiments did not observe the surface-site dependence of the $\mathrm{CO}$ lifetimes and the CO lifetime on the step atop site determined then was too long $(\sim 7 \mathrm{ps})^{10,24}$. The CO relaxation on larger particle sizes in this work showed clear site dependence. The energy dissipation is slightly faster on the bridge site than the terrace atop site, and it is faster on the step atop site than the terrace atop site. According to previous theoretical study ${ }^{27}$, vibrational relaxations induced by electron/vibration coupling are determined not only by the available electronic energy levels but also by the coupling strength between the surface electrons and the chemical bond that vibrates. The available energy levels of surface electrons are identical for each surface site. And, thus, the experimental results suggest that the $\mathrm{CO} /$ surface electron coupling strengths vary on different surface sites of nanoparticle, consistent with the different binding strength of $\mathrm{CO}$ molecules on distinct surface sites from previous studies on Pt flat surface ${ }^{26,47}$.

Particle-size and surface-site dependences on Pd surfaces. Similar trends are also observed on the surfaces of Pd nanoparticles. As displayed in Fig. 5d, the $\mathrm{CO}$ stretch vibrational relaxation $(3.5 \pm 0.5 \mathrm{ps})$ on the step atop site of $5 \mathrm{~nm}$ Pd metallic particles is about 20 times faster than that $(67 \pm 15 \mathrm{ps})$ on the atop site of $\sim 1 \mathrm{~nm} \mathrm{Pd} \mathrm{semi-conductive} \mathrm{clusters} \mathrm{(} \mathrm{Pd} / \mathrm{Ag}$ bimetallic particle $<2 \mathrm{~nm}$ ). The relaxation times follow the same relative order as those of metallic Pd particles: bridge $(2.0 \pm 0.2 \mathrm{ps})$ $<$ step atop $(3.5 \pm 0.5 \mathrm{ps})<$ terrace atop $(6.1 \pm 0.5 \mathrm{ps})(\ll 1 \mathrm{~nm}$ $(67 \pm 15 \mathrm{ps})$ ) (Fig. 5e). Comparison of the $5 \mathrm{~nm}$ Pd to the $5 \mathrm{~nm} \mathrm{Pt}$ shows the CO stretch vibrational relaxation on Pd is slightly slower than that on $\mathrm{Pt}$ at each surface site. This is probably because $\mathrm{CO} / \mathrm{Pt}$ interactions are slightly stronger than $\mathrm{CO} / \mathrm{Pd}$ interactions ${ }^{48}$.

\section{Discussion}

Two distinct energy migration pathways on nanoparticle surfaces were observed. On a large metallic nanoparticle, the vibrational energy of a surface molecule can rapidly dissipate into the particle through electron/hole pair excitations. The electron/hole pairs can then quickly recombine and release their energy to the lattice motions (phonons, or 'heat'), and quickly migrate on the surface, raising the surface temperature. All the processes occur within a few picoseconds. On a small and semiconducting particle, because of the lack of electron/vibration coupling, the surface vibrational relaxation and the energy migration between surface molecules are mainly through the vibration/vibration coupling mechanism and are $>1$ order of magnitude slower. Systematic studies also reveal that the electron/vibration coupling is little dependent on temperature (from 80 to $293 \mathrm{~K}$ ) or the particle size only if the particle is metallic, but is dependent on the particle surface site and the nature of the metal. The findings may stimulate further application and development of existing nonadiabatic theory to explore the dependences ${ }^{23}$.

\section{Methods}

Materials and synthesis procedures. The chemicals used for synthesizing the nanoparticles were purchased from Sigma-Aldrich and used without further purification and the $\mathrm{CO}$ gas was purchased from Matheson and Sigma.

Preparation and characterization of $1 \mathbf{~ n m ~ P t ~ n a n o p a r t i c l e . ~ A b o u t ~} 0.5 \mathrm{ml}(0.1 \mathrm{M})$ aqueous solution of chloroplatinic acid $\left(\mathrm{H}_{2} \mathrm{PtCl}_{6} \cdot 6 \mathrm{H}_{2} \mathrm{O}\right)$ dissolved in $10 \mathrm{ml}$ dimethylformamide (DMF) can be sealed into $1.5 \mathrm{~atm}$ CO glass vessel for $28 \mathrm{~h}$ to obtain the blue-green sample. The structure of the cluster was characterized with mass spectrometry ${ }^{43}$. In the DMF solution (Fig. $1 \mathrm{~b}$ and Supplementary Fig. 1a), the atop site $\mathrm{CO}$ absorption is at $2,047 \mathrm{~cm}^{-1}$, and the bridge site $\mathrm{CO}$ absorption is around $1,860 \mathrm{~cm}^{-1}$. The FTIR spectrum in Fig. 1c multiplies the intensities of the atop $\mathrm{CO}$ and bridge $\mathrm{CO}$ absorption regions by 25 times for better illustration. The IR spectrum (Supplementary Fig. 1c) showed in solid phase also confirmed two main peaks of this sample. The $1 \mathrm{~nm}$ Pt particles are unstable in the solid state. All ultrafast measurements presented in this paper were conducted in the DMF solution and finished within $2-3 \mathrm{~h}$. The ultraviolet-visible spectra of the reaction solution before and after the reaction were recorded using a Varian Cary 5000 UV-visible spectrometer. Compared with the $\mathrm{H}_{2} \mathrm{PtCl}_{6}$ solution, two new peaks (Supplementary Fig. 1d) at 400 and $630 \mathrm{~nm}$ appear in the cluster solution, consistent with those of the $\left[\mathrm{Pt}_{3}(\mathrm{CO})_{3}\left(\mu_{2}-\mathrm{CO}\right)_{3}\right]_{5}^{2-}$. The $1.7 \mathrm{eV}$ energy gap is calculated according to the ultraviolet-visible spectrum because this sample's electronic properties are comparable to a quantum dot.

\section{Preparation and characterization of $\mathbf{2} \mathbf{n m}$ Pt nanoparticle solid sample.}

$\mathrm{K}_{2} \mathrm{PtCl}_{4}(3 \mathrm{mM}$ ethylene glycol solution) and $10 \mathrm{mM}$ sodium acetate were mixed in hot 1, 2-ethanediol at $80^{\circ} \mathrm{C}$, stirred for $30 \mathrm{~min}$. Thus, $\sim 2 \mathrm{~nm}$ Pt nanoparticles were synthesized following the reported method ${ }^{49}$. The hot solution was then cooled down. $\mathrm{CO}$ gas was bubbled at the rate of $200 \mathrm{ml} \mathrm{min}^{-1}$ within $30 \mathrm{~min}$ in a hood. The solution was then mixed with water at a v/v ratio $3: 1$. The mixture was centrifuged for three times with 26,000 r.p.m. for $38 \mathrm{~h}$ or 40,000 r.p.m. for $20 \mathrm{~h}$. The precipitate was suspended in several drops of 1,2-ethanediol. The mixture was dropped onto $\mathrm{CaF}_{2}$ windows and transferred into the vacuum oven overnight to remove solvent. The sample-covered $\mathrm{CaF}_{2}$ window was placed into vacuum chamber and oil-free pumped overnight. We applied the same procedures to bubble Pt nanoparticles (2, 5 and $11 \mathrm{~nm}$ ) and Pd nanoparticles ( 1 and $5 \mathrm{~nm}$ ) samples, prepared the solid samples in vacuum chamber, measure the solid samples FTIR spectrum and collect ultrafast data. The frequency ranges $(1,830-$ $\left.1,890 \mathrm{~cm}^{-1}\right)$ and $\left(2,090-2,130 \mathrm{~cm}^{-1}\right)$ of FTIR Fig. $1 \mathrm{c}$ of $2 \mathrm{~nm}$ sample in the result part were zoomed in three times for better illustration. The sample was characterized by JEM 2100F TEM and Rigaku D/Max Ultima XRD. The transmission electron microscopy (TEM) and XRD data are shown in Supplementary Fig. 2a,b (high resolution) and Supplementary Fig. 3a. The temperature control measurements were performed with JANIS model ST-100.

\section{Preparation and characterization of $\mathbf{2} \mathbf{~ n m ~ P t ~ n a n o p a r t i c l e ~ s o l u t i o n ~ s a m p l e . ~}$} $\mathrm{K}_{2} \mathrm{PtCl}_{4}\left(0.0174 \mathrm{~g}, 4.2 \times 10^{-5} \mathrm{~mol}\right)$, poly(acrylic acid sodium salt) (molecular weight $(\mathrm{MW}) \sim 5,100,0.0394 \mathrm{~g}$ ) and $10 \mathrm{ml}$ ethylene glycol were mixed in a $50 \mathrm{ml}$ flask. It is noted that poly(acrylic acid sodium salt) shows poor solubility in ethylene glycol at room temperature. Under vigorously stirring, the solution was heated to $180^{\circ} \mathrm{C}$ and kept at $180^{\circ} \mathrm{C}$ for $10 \mathrm{~min}$. The colour of solution would become dark and clear with increasing temperature, which indicates the formation of $\sim 2-3 \mathrm{~nm}$ Pt NPs. Then, the sample was dropped on the $\mathrm{CaF}_{2}$ window. Vacuum pump was used to remove main parts of ethylene glycol solvents in the vacuum oven. Only two drops of high-concentration solution were left on the $\mathrm{CaF}_{2}$ window. Another $\mathrm{CaF}_{2}$ was used to quickly seal the sample between two $\mathrm{CaF}_{2}$. This solution sample did not need to be transferred into vacuum chamber for measurement. The FTIR is shown in Supplementary Fig. 3b. The method to prepare isotope-labelled $2 \mathrm{~nm} \mathrm{Pt}$ was exactly same as that for the powder $2 \mathrm{~nm} \mathrm{Pt}$ NP sample. $\mathrm{K}_{2} \mathrm{PtCl}_{4}(3 \mathrm{mM}$ ethylene glycol solution) and $10 \mathrm{mM}$ sodium acetate were mixed in hot 1,2 -ethanediol at $80^{\circ} \mathrm{C}$, stirred for $30 \mathrm{~min}$. The isotope ${ }^{13} \mathrm{C}^{18} \mathrm{O}$ gas 0.51 (purchased from Sigma) was bubbled into the solution. Samples were then prepared according to the procedure described above.

FTIR spectra of $\mathrm{CO}$ and ${ }^{13} \mathrm{C}^{18} \mathrm{O}$ on the $2 \mathrm{~nm}$ Pt nanoparticles were shown in Supplementary Fig. 3c. The vibrational decay data of the ${ }^{13} \mathrm{C}^{18} \mathrm{O}$ stretch excitation in this paper were obtained by exciting the shoulder at $\sim 1,940 \mathrm{~cm}^{-1}$. The 2D IR of this sample is shown in Supplementary Fig. 3d. The blue peak around $\left(1940 \mathrm{~cm}^{-1}, 1870 \mathrm{~cm}^{-1}\right)$ stems from the ${ }^{13} \mathrm{C}^{18} \mathrm{O} 1-2$ transition and indicates its binding formation on the step site.

Preparation and characterization of $\mathbf{5} \mathbf{~ n m ~ P t ~ n a n o p a r t i c l e . ~ P t ( I I ) ~ a c e t y l a c e t o n a t e ~}$ $(80 \mathrm{mg}, \sim 0.2 \mathrm{mmol}$ ) and polyvinylpyrrolidone (PVP, $55 \mathrm{mg}, \mathrm{MW}=55,000)$ were dissolved in $5 \mathrm{ml}$ of ethylene glycol. The solution was then heated to $200^{\circ} \mathrm{C}$ in a microwave reactor (Anton Paar Monowave 300) under a stirring rate of 1200 r.p.m. $\mathrm{min}^{-1}$ and held at that temperature for $5 \mathrm{~min}$ and then cooled to room temperature. The as-prepared Pt nanoparticles were precipitated with $45 \mathrm{ml}$ of acetone and re-dispersed in $10 \mathrm{ml}$ of ethylene glycol to remove excess PVP. Thus, the $5 \mathrm{~nm}$ Pt nanoparticles were synthesized according to the reported method ${ }^{50}$. The TEM image is shown in Supplementary Fig. 2c. The original FTIR intensities of the bridge and terrace are weak. In the Results part, FTIR Fig. 1c bridge area $\left(1,830-1,890 \mathrm{~cm}^{-1}\right)$ and terrace area $\left(2,090-2,130 \mathrm{~cm}^{-1}\right)$ of $5 \mathrm{~nm}$ sample were zoomed in 10 times for better illustration.

Preparation and characterization of $\mathbf{1 1} \mathbf{~ n m ~ P t ~ n a n o p a r t i c l e . ~ F i r s t , ~} 250 \mathrm{ml}$ $1 \times 10^{-4} \mathrm{M} \mathrm{K}_{2} \mathrm{PtCl}_{4}$ solution in DI water was prepared, followed by adding $0.2 \mathrm{ml}$ of $0.1 \mathrm{M}$ sodium polyacrylate. Then $\mathrm{N}_{2}$ gas (>99.99\%, Matheson) was bubbled into the mixture at a flow rate of $200 \mathrm{ml} \mathrm{min}^{-1}$ for $20 \mathrm{~min}$. Reduction of Pt ions was started by switching the bubbling gas to $\mathrm{H}_{2}(>99.99 \%$, Matheson) for $10 \mathrm{~min}$ at a flow rate of $200 \mathrm{ml} \mathrm{min}^{-1}$. The reaction vessel was then sealed and left under room 
temperature for $48 \mathrm{~h}$ to complete the reduction. Thus, the preparation method of the $11 \mathrm{~nm}$ Pt nanoparticles in this study followed the method by Ahmadi et al. ${ }^{51}$ The TEM picture is shown in Supplementary Fig. $2 \mathrm{~d}$.

Preparation and characterization of $\mathbf{5} \mathbf{~ n m ~ P d}$ nanoparticle. About 0.449 $(2.00 \mathrm{mmol})$ of palladium(II) acetate and $0.85 \mathrm{~g}$ of PVP $(\mathrm{MW}=55,000)$ were dissolved in $20 \mathrm{ml}$ of 2-ethoxyethanol. The solution was then heated to $110^{\circ} \mathrm{C}$ in a microwave reactor (Anton Paar Monowave 300) under a stirring rate of 1,200 r.p.m. $\min ^{-1}$ and held at that temperature for $60 \mathrm{~min}$, and then cooled to room temperature. The as-prepared Pd NPs are used for spectroscopic measurement. The TEM picture is shown in Supplementary Fig. 2e. The FTIR of this sample is shown in Supplementary Fig. 4.

Preparation and characterization of $\mathbf{1} \mathbf{~ m ~ P d - A g ~ n a n o p a r t i c l e . ~ T h e ~ p r e p a r a t i o n ~}$ method was a revised method for the preparation of $\mathrm{Ag}_{44}$ (ref. 52). Supplementary Fig. $5 \mathrm{a}$ briefly presented the synthesis procedure. In detail, $0.25 \mathrm{mmol}$ of silver nitrate $\left(\mathrm{AgNO}_{3}\right)$ and $0.0625 \mathrm{mmol}$ of palladium acetate $\left(\mathrm{Pd}\left(\mathrm{O}_{2} \mathrm{CCH} 3\right)_{2}\right)$ were dissolved in $21 \mathrm{ml}$ water and $1.25 \mathrm{mmol}$ of para-mercaptobenzoic acid (p-MPA) were dissolved in $12 \mathrm{ml}$ ethanol. The aqueous solution and ethanolic solution were mixed to form a yellowish insoluble precursor. The $\mathrm{pH}$ was then raised to 12 to deprotonate $\mathrm{p}$-MPA by using aqueous $\mathrm{CsOH}(50 \% \mathrm{w} / \mathrm{v})$. The colour of solution immediately became red with the increase of $\mathrm{pH}$ value. After that $9 \mathrm{ml}$ of aqueous $\mathrm{NaBH}_{4}(3.125 \mathrm{mmol})$ was slowly added to the precursor solution with vigorously stirring. The colour of solution turned to dark after adding $\mathrm{NaBH}_{4}$ solution. After $1 \mathrm{~h}$ reaction, the obtained product was cleaned first by centrifuging to remove any solid and then precipitating the clusters with DMF to remove salts and other leftover soluble materials from the reaction. TEM picture is shown in Supplementary Fig. 5b and X-ray photoelectron spectroscopy (XPS) is shown in Supplementary Fig. $5 c$. The ratio of $\mathrm{Pd} / \mathrm{Ag} / \mathrm{S}$ equals 1:6.2:6.8, calculated from the peak area/relatively sensitivity factor with the software Multipak of the XPS machine. Thus, the as-prepared nanoparticle is the $\mathrm{Pd}-\mathrm{Ag}$ bimetallic structure. The low metal/ligand ratio suggested the small size of this particle. The multi-absorbance peaks in ultraviolet-visible spectrum also indicate that the size of $\mathrm{Pd}-\mathrm{Ag}$ bimetallic nanoparticles is $<2 \mathrm{~nm}$-otherwise, a strong surface Plasmon resonance peak from $\mathrm{Ag}$ nanoparticles would be observed at $\sim 420 \mathrm{~nm}$ (ref. 53). The TEM image (Supplementary fig. 5b) also suggests that the size is very small $(<2 \mathrm{~nm})$ and results in poor image resolution. Because the binding strength of $\mathrm{CO}$ with $\mathrm{Pd}$ is much stronger than that of $\mathrm{CO}$ with $\mathrm{Ag}$ (refs 54,55), the $\mathrm{CO}$ molecules on the $\mathrm{Ag}$ $\mathrm{Pd}$ bimetallic nanoparticle dominantly reside on Pd atoms.

Optical methods. Briefly, the same seed pulse is employed to synchronize a picosecond amplifier and a femtosecond amplifier. The two amplifiers are then used to produce pump and probe IR beams, respectively. The $\sim 0.8 \mathrm{ps}$ mid-IR pulses (vary from 0.7 to $0.9 \mathrm{ps}$ according to the frequency) were generated by the ps amplifier pumping an optical parametric amplifier. The bandwidth of the $1 \mathrm{kHz}$ mid-IR beam as pumping beam in measurement was around $10-35 \mathrm{~cm}^{-1}$ in a tunable frequency range of 400 to $4,000 \mathrm{~cm}^{-1}$ with energy ranging from $1 \sim 40 \mu \mathrm{J}$ per pulse $\left(>10 \mu \mathrm{J}\right.$ per pulse for $\left.1,000-4,000 \mathrm{~cm}^{-1}\right)$. The probing beam (a highintensity mid-IR and terahertz super-continuum pulse with a duration of $<100 \mathrm{fs}$ between 400 to $3,000 \mathrm{~cm}^{-1}$ at $1 \mathrm{KHz}$ ) was generated from the fematosecond amplifier. Two polarizers are inserted into the probe beam pathway before and after the sample, respectively, to selectively measure the parallel or perpendicular polarized signal relative to the excitation beam. In IR pump/IR probe experiments, the picosecond IR pulse is the pump beam and the super-continuum pulse is the probe beam. Two polarizers are inserted into the probe beam pathway to selectively measure the parallel or perpendicular polarized signal relative to the excitation beam. To avoid the scattering signal (especially for the weak signals at the bridge site and terrace atop site), we used data from the perpendicular configuration rather than the usual rotation-free data to describe the vibrational decay ${ }^{56}$. This approach can introduce some uncertainty in the vibrational lifetimes as resonant vibrational energy transfers and rotation can occur. To address this issue, for both 1 and $5 \mathrm{~nm}$ Pt samples, we measured both rotation free (parallel signal plus two perpendicular signals) and perpendicular signals. The data are plotted in Supplementary Fig. 11 and show that the decays from the perpendicular measurements are about $10 \%$ slower than the rotation-free data. Because our experimental data have $\pm 10 \%$ uncertainty, the data of the isotope labelling ${ }^{13} \mathrm{C}^{18} \mathrm{O}$, the data do not present a clear difference with the un-labelled data. In all our 2D IR spectra in this paper, the probe frequency resolution is around $\pm 4 \mathrm{~cm}^{-1}$ and the pump frequency resolution is $\sim 20 \mathrm{~cm}^{-1}$. The FTIR spectral resolution is around $2 \mathrm{~cm}^{-1}$. The control experiment of heat generation of peak 2 in Figs 2 and 3 is introduced in Supplementary Fig. 12. Our signal origins of both diagonal peaks and cross peaks are presented in Supplementary Method.

\section{References}

1. Wodtke, A. M., Tully, J. C. \& Auerbach, D. J. Electronically non-adiabatic interactions of molecules at metal surfaces: Can we trust the BornOppenheimer approximation for surface chemistry? Inter. Rev. Phys. Chem. 23, 513-539 (2004).
2. Tully, J. C. Chemical dynamics at metal surfaces. Ann. Rev. Phys. Chem. 51, 153-178 (2000).

3. Wodtke, A. M., Matsiev, D. \& Auerbach, D. J. Energy transfer and chemical dynamics at solid surfaces: The special role of charge transfer. Prog. Surf. Sci. 83, 167-214 (2008).

4. Jaeger, N. I. Bridging gaps and opening windows. Science 293, 1601-1602 (2001).

5. Rosenfeld, D. E., Gengeliczki, Z., Smith, B. J., Stack, T. \& Fayer, M. Structural dynamics of a catalytic monolayer probed by ultrafast $2 \mathrm{D}$ IR vibrational echoes. Science 334, 634-639 (2011).

6. Forsblom, M. \& Persson, M. Vibrational lifetimes of cyanide and carbon monoxide on noble and transition metal surfaces. J. Chem. Phys. 127, 154303 (2007).

7. Nieto, P. et al. Reactive and nonreactive scattering of $\mathrm{H} 2$ from a metal surface is electronically adiabatic. Science 312, 86-89 (2006).

8. Díaz, C. et al. Multidimensional effects on dissociation of $\mathrm{N}_{2}$ on $\mathrm{Ru}(0001)$. Phys. Rev. Lett. 96, 096102 (2006).

9. Harris, A. \& Rothberg, L. Surface vibrational energy relaxation by sum frequency generation: five-wave mixing and coherent transients. J. Chem. Phys. 94, 2449-2457 (1991).

10. Berkerle, J., Casassa, M., Cavanagh, R., Heilweil, E. \& Stephenson, J. Time resolved studies of vibrational relaxation dynamics of $\mathrm{CO}(\mathrm{v}=1)$ on metal particle surfaces. J. Chem. Phys. 90, 4619-4620 (1989).

11. Hasselbrink, E. Capturing the complexities of molecule-surface interactions. Science 326, 809-810 (2009).

12. Wodtke, A. M. Chemistry in a computer: advancing the in silico dream. Science 312, 64-65 (2006).

13. Hurst, J. E., Wharton, L., Janda, K. C. \& Auerbach, D. J. Direct inelastic scattering Ar from Pt (111). J. Chem. Phys. 78, 1559-1581 (1983).

14. Hurst, J. E. et al. Observation of direct inelastic scattering in the presence of trapping-desorption scattering: Xe on Pt (111). Phys. Rev. Lett. 43, 1175 (1979).

15. Tully, J. C. Theories of the dynamics of inelastic and reactive processes at surfaces. Annu. Rev. Phys. Chem. 31, 319-343 (1980).

16. Auerbach, D. J. Hitting the surface--softly. Science 294, 2488-2489 (2001).

17. Gergen, B., Nienhaus, H., Weinberg, W. H. \& McFarland, E. W. Chemically induced electronic excitations at metal surfaces. Science 294, 2521-2523 (2001).

18. Huang, Y., Rettner, C. T., Auerbach, D. J. \& Wodtke, A. M. Vibrational promotion of electron transfer. Science 290, 111-114 (2000).

19. Hou, H. et al. Enhanced reactivity of highly vibrationally excited molecules on metal surfaces. Science 284, 1647-1650 (1999).

20. White, J. D., Chen, J., Matsiev, D., Auerbach, D. J. \& Wodtke, A. M. Conversion of large-amplitude vibration to electron excitation at a metal surface. Nature 433, 503-505 (2005).

21. Kroes, G.-J., Díaz, C., Pijper, E., Olsen, R. A. \& Auerbach, D. J. Apparent failure of the Born-Oppenheimer static surface model for vibrational excitation of molecular hydrogen on copper. Proc. Natl Acad. Sci. USA 107, 20881-20886 (2010).

22. Beckerle, J. D., Casassa, M. P., Cavanagh, R. R., Heilweil, E. J. \& Stephenson, J. C. Ultrafast infrared response of adsorbates on metal surfaces: vibrational lifetime of CO/Pt(111). Phys. Rev. Lett. 64, 2090-2093 (1990).

23. Shenvi, N., Roy, S. \& Tully, J. C. Dynamical steering and electronic excitation in NO scattering from a gold surface. Science 326, 829-832 (2009).

24. Heilweil, E., Cavanagh, R. \& Stephenson, J. Picosecond study of the population lifetime of $\mathrm{CO}(\mathrm{v}=1)$ chemisorbed on $\mathrm{SiO}_{2}$-supported rhodium particles. J. Chem. Phys. 89, 5342-5343 (1988).

25. Guasco, T. L., Elliott, B. M., Johnson, M. A., Ding, J. \& Jordan, K. D. Isolating the spectral signatures of individual sites in water networks using vibrational double-resonance spectroscopy of cluster isotopomers. J. Phys. Chem. Lett. 1, 2396-2401 (2010).

26. Backus, E. H. G., Forsblom, M., Persson, M. \& Bonn, M. Highly efficient ultrafast energy transfer into molecules at surface step sites. J. Phys. Chem. C 111, 6149-6153 (2007)

27. Persson, B. \& Persson, M. Vibrational lifetime for $\mathrm{CO}$ adsorbed on $\mathrm{Cu}(100)$ Solid State Commun. 36, 175-179 (1980).

28. Wang, Z., Ahmad, T. \& El-Sayed, M. Steps, ledges and kinks on the surfaces of platinum nanoparticles of different shapes. Surf. Sci. 380, 302-310 (1997).

29. Tripa, C. E. \& Yates, J. T. Surface-aligned reaction of photogenerated oxygen atoms with carbon monoxide targets. Nature 398, 591-593 (1999).

30. Longoni, G. \& Chini, P. Synthesis and chemical characterization of platinum carbonyl dianions [Pt3 (CO) 6] $\mathrm{n} 2$-( $\mathrm{n}=$. apprx. 10, 6, 5, 4, 3, 2, 1). A new series of inorganic oligomers. J. Am. Chem. Soc. 98, 7225-7231 (1976).

31. Hayden, B. E. \& Bradshaw, A. M. The adsorption of CO on Pt(111) studied by infrared reflection-Absortion spectroscopy. Surf. Sci. 125, 787-802 (1983).

32. Lundwall, M. J., McClure, S. M. \& Goodman, D. W. Probing terrace and step sites on Pt nanoparticles using CO and ethylene. J. Phys. Chem. C 114, 7904-7912 (2010).

33. Golonzka, O., Khalil, M., Demirdoven, N. \& Tokmakoff, A. Vibrational anharmonicities revealed by coherent two-dimensional infrared spectroscopy. Phys. Rev. Lett. 86, 2154-2157 (2001). 
34. Cervetto, V., Helbing, J., Bredenbeck, J. \& Hamm, P. Double-resonance versus pulsed Fourier transform two-dimensional infrared spectroscopy: an experimental and theoretical comparison. J. Chem. Phys. 121, 5935-5942 (2004).

35. Zheng, J. et al. Ultrafast dynamics of solute-solvent complexation observed at thermal equilibrium in real time. Science 309, 1338-1343 (2005).

36. Chen, H., Bian, H., Li, J., Wen, X. \& Zheng, J. Relative intermolecular orientation probed via molecular heat transport. J. Phys. Chem. A 117, 6052-6065 (2013).

37. Chen, H., Bian, H., Li, J., Wen, X. \& Zheng, J. Ultrafast multiple-mode multiple-dimensional vibrational spectroscopy. Inter. Rev. Phys. Chem. 31, 469-565 (2012).

38. Yau, S. H., Varnavski, O. \& Goodson, III T. An ultrafast look at Au nanoclusters. Acc. Chem. Res. 46, 1506-1516 (2013).

39. Schmid, G. Large clusters and colloids. Metals in the embryonic state. Chem. Rev. 92, 1709-1727 (1992).

40. Li, L. et al. Investigation of catalytic finite-size-effects of platinum metal clusters. J. Phys. Chem. Lett. 4, 222-226 (2012).

41. Wang, Z. et al. Ultrafast flash thermal conductance of molecular chains. Science 317, 787-790 (2007).

42. Frischkorn, C. \& Wolf, M. Femtochemistry at metal surfaces: nonadiabatic reaction dynamics. Chem. Rev. 106, 4207-4233 (2006).

43. Chen, G., Yang, H., Wu, B., Zheng, Y. \& Zheng, N. Supported monodisperse Pt nanoparticles from $\left[\mathrm{Pt} 3(\mathrm{CO})_{3}(\mu 2-\mathrm{CO})_{3}\right]$ 52-clusters for investigating support-Pt interface effect in catalysis. Dalton Trans. 42, 12699-12705 (2013).

44. Selvakannan, P., Lampre, I., Erard, M. \& Remita, H. Platinum carbonyl clusters: double emitting quantum dots. J. Phys. Chem. C 112, 18722-18726 (2008).

45. Chen, H., Wen, X., Guo, X. \& Zheng, J. Intermolecular vibrational energy transfers in liquids and solids. Phys. Chem. Chem. Phys. 16, 13995-14014 (2014).

46. Heilweil, E., Stephenson, J. \& Cavanagh, R. Measurements of carbonyl ( $\mathrm{v}=1)$ population lifetimes: metal-carbonyl cluster compounds supported on silica. J. Phys. Chem. 92, 6099-6103 (1988).

47. Backus, E. H., Eichler, A., Kleyn, A. W. \& Bonn, M. Real-time observation of molecular motion on a surface. Science 310, 1790-1793 (2005).

48. Hammer, B., Morikawa, Y. \& Nørskov, J. K. CO chemisorption at metal surfaces and overlayers. Phys. Rev. Lett. 76, 2141 (1996).

49. Dablemont, C. et al. FTIR and XPS study of Pt nanoparticle functionalization and interaction with alumina. Langmuir 24, 5832-5841 (2008).

50. Wang, H. et al. Influence of size-induced oxidation state of platinum nanoparticles on selectivity and activity in catalytic methanol oxidation in the gas phase. Nano Lett. 13, 2976-2979 (2013).

51. Ahmadi, T. S., Wang, Z. L., Green, T. C., Henglein, A. \& El-Sayed, M. A. Shape-controlled synthesis of colloidal platinum nanoparticles. Science 272, 1924-1925 (1996).

52. Desireddy, A. et al. Ultrastable silver nanoparticles. Nature 501, 399-402 (2013).

53. Peng, S., McMahon, J. M., Schatz, G. C., Gray, S. K. \& Sun, Y. Reversing the size-dependence of surface plasmon resonances. Proc. Natl Acad. Sci. USA 107, 14530-14534 (2010)
54. Zeinalipour-Yazdi, C. D., Cooksy, A. L. \& Efstathiou, A. M. CO adsorption on transition metal clusters: trends from density functional theory. Surf. Sci. 602, 1858-1862 (2008).

55. Zhao, S., Ren, Y., Ren, Y., Wang, J. \& Yin, W. Density functional study of CO binding on small $\mathrm{Ag}<\mathrm{i}>\mathrm{n}</ \mathrm{i}>\mathrm{Pd}<\mathrm{i}>\mathrm{m}</ \mathrm{i}>$ clusters. J. Mol. Struct. 955, 66-70 (2010).

56. Bian, H. et al. Molecular conformations and dynamics on surfaces of gold nanoparticles probed with multiple-mode multiple-dimensional infrared spectroscopy. J. Phys. Chem. C 116, 7913-7924 (2012).

57. Zheng, J. et al. Accidental vibrational degeneracy in vibrational excited states observed with ultrafast Two-Dimentional IR vibrational echo spectroscopy. J. Chem. Phys. 123, 164301 (2005).

\section{Acknowledgements}

This material is based on the work supported by the AFOSR Award No. FA9550-11-1 0070 and the Welch foundation under Award No. C-1752. J.Z. also thanks supports from PKU and the David and Lucile Packard Foundation for a Packard fellowship and the Alfred P. Sloan Foundation for a Sloan fellowship. N.Z. appreciates the funding from the National Nature Science Foundation of China (21131005, 21420102001). M.S.W. acknowledges J. Evans Attwell-Welch Postdoctoral Fellowship Program of the Smalley Institute of Rice University (to H.Q.) and the National Science Foundation (CBET-1134535).

\section{Author contributions}

J.Z. and J.L. designed the experiments. J.Z. supervised the project. J.L., H.C., K.Y., X.G. and A.M. performed ultrafast experiments. H.Q., J.L., Z.Z., X.G., N.Z. and M.S.W. conducted the synthesis experiments. Y.C. performed $1 \mathrm{~nm}$ Pt sample temperaturedependent FTIR. J.Z., J.L., H.Q. and A.M. prepared and revised the manuscript.

\section{Additional information}

Supplementary Information accompanies this paper at http://www.nature.com/ naturecommunications

Competing financial interests: The authors declare no competing financial interests.

Reprints and permission information is available online at http://npg.nature.com/ reprintsandpermissions/

How to cite this article: Li, J. et al. Two distinctive energy migration pathways of monolayer molecules on metal nanoparticle surfaces. Nat. Commun. 7:10749 doi: 10.1038/ncomms10749 (2016).

This work is licensed under a Creative Commons Attribution 4.0 International License. The images or other third party material in this article are included in the article's Creative Commons license, unless indicated otherwise in the credit line; if the material is not included under the Creative Commons license, users will need to obtain permission from the license holder to reproduce the material. To view a copy of this license, visit http://creativecommons.org/licenses/by/4.0/ 